\title{
Continuous subpalpebral antimicrobial lavage for pseudomonas scleritis secondary to pterygium excision
}

\author{
Colin I. Clement, ${ }^{1,2}$ Margaret Kearns, ${ }^{3}$ Paula Berdoukas, ${ }^{1,3}$ \\ Peter McCluskey ${ }^{1,2,3,4}$ \\ 'Department of Ophthalmology, Sydney Hospital \& Sydney Eye Hospital, Sydney \\ NSW 2000, Australia; \\ ${ }^{2}$ Central Clinical School, The University of Sydney, Sydney NSW 2000 Australia; \\ ${ }^{3}$ Department of Ophthalmology, Royal Prince Alfred Hospital, Camperdown NSW \\ 2006 Australia; \\ ${ }^{4}$ Save Sight Institute, Sydney NSW 2000 Australia
}

Keywords: Scleritis, pseudomonas, pterygium, ciprofloxacin

\section{Case report}

Infectious scleritis is an uncommon but potentially blinding complication of pterygium surgery. The use of ß-irradiation at the time of surgery is a strong risk factor for this complication. ${ }^{1}$ Up to $81 \%$ of cases are caused by Pseudomonas aeruginosa. $^{2}$

Treatment remains the main challenge in this condition. Despite the delivery of appropriate antibiotics and the use of surgical debridement, outcomes remain variable and unpredictable with a significant number of cases resulting in enucleation or eye salvage with visual acuity worse than $20 / 200 .^{3}$

Subpalpebral antimicrobial lavage has been reported for the management of infectious scleritis with encouraging results. A series of six cases of infectious scleritis, including two cases of pseudomonas scleritis following pterygium excision, showed significant visual improvement following this treatment. However, despite this, the technique is not widely used. We report here a case of pseudomonas scleritis that was treated successfully with subpalpebral antimicrobial lavage in an attempt to increase awareness of this management option.

In June 2008, a 74 year-old female presented for further management of a right progressive infectious necrotizing scleritis (Fig. 1a). She had undergone right medial pterygium excision with $\beta$-irradiation in 1985. A swab of the involved sclera was culture positive for pseudomonas aeruginosa (ciprofloxacin-sensitive). At the time of presentation, her BCVA was OD 6/24 OS 6/6 with scleritis progressing despite topical q1h ciprofloxacin $0.3 \%$, a subconjunctival bolus of gentamicin $0.9 \%$ and oral

\section{Correspondence:}

Dr Colin Clement, Department of Ophthalmology, Sydney Hospital \& Sydney Eye Hospital, 8 Macquarie Street, Sydney, NSW, AUSTRALIA 2000.

E-mail: colinc1@med.usyd.edu.au 


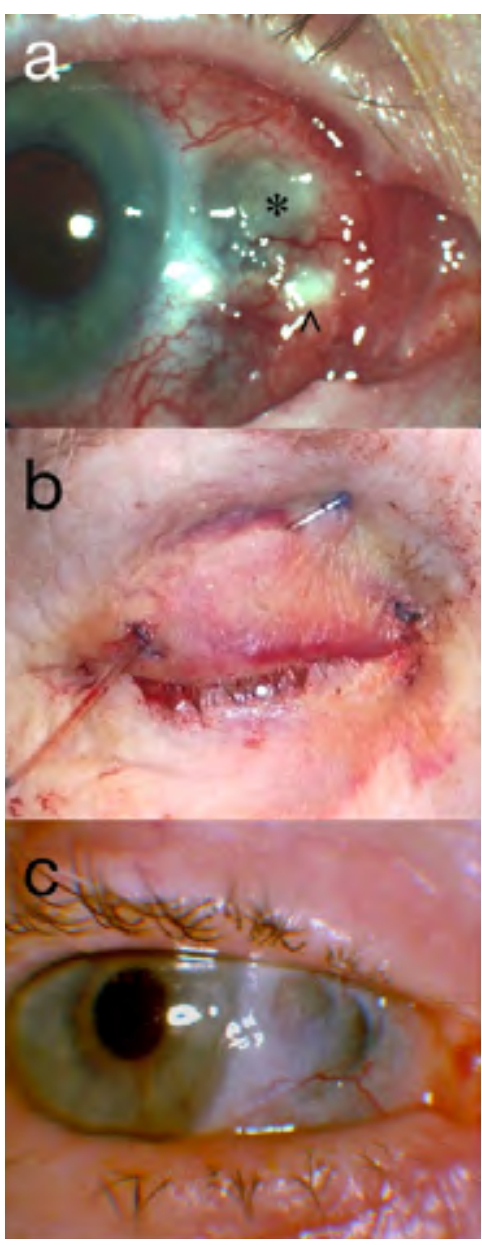

Figure 1.a) patients right eye at presentation demonstrating medial scleral necrosis $\left({ }^{*}\right)$ with inferior abscess $(\wedge)$, b) sub-palpebral lavage catheter in situ for continuous antimicrobial lavage, c) the same eye $\mathbf{2 6}$ months after presentation. ciprofloxacin $500 \mathrm{mg}$ BD over the preceding four weeks. The patient was admitted to hospital and commenced on ticarcillin 3.1gm QID ivi and continued on topical q1h ciprofloxacin $0.3 \%$. Forty-eight hours after admission, the patient was taken to theatre where a 360-degree conjunctival peritomy was performed. Scleral abscesses were identified inferomedially and inferolaterally. Repeat swabbing at this time was culture negative. Gentle debridement followed by subconjunctival washout with gentamicin $0.9 \%$ was performed. A fenestrated gortex epidural catheter was positioned in the superior fornix via the upper lid using a trochar and secured with 26.0 prolene skin sutures (Fig. 2). An infusion of ciprofloxacin $200 \mathrm{mg} / 100 \mathrm{ml}$ was connected to the catheter and commenced at $15 \mathrm{ml} / \mathrm{hr}$ using a volumetric infusion pump. Continuous infusion was maintained for the first 24 hours then restricted to $0600-2200$ for the following five days. By day 3 of lavage treatment, the entire ocular surface was covered in ciprofloxacin precipitates. Therefore, from day 3 to the end of lavage treatment, daily manual debridement of the deposits was performed followed by the application of a $15-\mathrm{mm}$ bandage contact lens. On day 9 (Fig. 1b), the patient was discharged home on $\mathrm{g}$. ofloxacin QID OD, g. bion tears $q 1 \mathrm{~h} O D$, g. prednefrin forte QID OD and oral ciprofloxacin $500 \mathrm{mg}$ BD.

Despite complete scleritis resolution, unaided VA remained $6 / 60$ due to increased axial length (from $23.26 \mathrm{~mm}$ to $23.82 \mathrm{~mm}$ ) and increased mean keratometry (from $44.76 \mathrm{D}$ to $47.06 \mathrm{D}$ ) resulting in a refraction of $-5.25 /+2.00$ $x 25$ degrees (6/9). This was treated by insertion of a Rayner Sulcoflex Toric IOL $(-6.5 /+2.00 \times 25$ degrees). At the last postoperative visit, refraction was plano/+0.50 x 180 degrees (6/6).

In patients with infectious scleritis, organisms may remain sequestered within the deep scleral layers, not induce an adequate inflammatory response, respond poorly to antimicrobial treatment or recur following seemingly adequate treatment. Subpalpebral lavage partly addresses these problems by supplying topical antibiotics at levels much higher (ciprofloxacin 2000 times MIC90 for pseudomonas 
aeruginosa) $)^{4}$ than can be achieved with manual instillation. The continuous nature of the treatment may also wash necrotic debris from the ocular surface and physically reduce the bacterial load. Any issues with compliance or timing are obviously also addressed with this technique.

In our case, the development of ciprofloxacin deposits caused ocular discomfort and led to a significant epithelial defect. Both tobramycin and levofloxacin lavage have been used successfully for subpalpebral lavage of pseudomonas scleritis without ocular surface toxicity ${ }^{5}$ and may be alternatives if toxicity is a concern.

\section{References}

1. Mackenzie FD, Hirst LW, Kynaston B, Bain C. Recurrance rate and complications after beta irradiation for pterygia. Ophthalmol 1991;98:1776-1780.

2. Su C-Y, Tsai J-J, Chang Y-C, Lin C-P. Immunologic and clinical manifestations of infectious scleritis after pterygium excision. Cornea 2006;25;663-666.

3. Hsiao C-H, Chen JJY, Huang SCM, Ma H-K, Chen PYF, Tsai RJF. Intrascleral dissemination of infectious scleritis following pterygium excision. Br J Ophthalmol 1998;82;29-34.

4. Hoogkamp-Korstanje JAA. In-vitro activites of ciprofloxacin, levofloxacin, lomefloxacin, ofloxacin, pefloxacin, sparfloxacin and trovafloxacin against gram-positive and gram-negative pathogens from respiratory tract infections. J Antimicrobial Chemother 1997;40:427-431.

5. Meallet MA. Subpalpebral lavage antibiotic treatment for severe infectious scleritis and keratitis. Cornea 2006;25:159-163. 